\title{
Trends in Cable Magnetization and Persistent Currents During the Production of the Main Dipoles of the Large Hadron Collider
}

\author{
Boris Bellesia, Luca Bottura, Valeria Granata, Sandrine Le Naour, Luc Oberli, Stephane Sanfilippo, Claudio Santoni, \\ Walter Scandale, Nikolai Schwerg, Ezio Todesco, and Christine Vollinger
}

\begin{abstract}
The production of more than $60 \%$ of superconducting cables for the main dipoles of the Large Hadron Collider has been completed. The results of the measurements of cable magnetization and the dependence on the manufacturers are presented. The strand magnetization produces field errors that have been measured in a large number of dipoles (approximately 100 to date) tested in cold conditions. We examine here the correlation between the available magnetic measurements and the large database of cable magnetization. The analysis is based on models documented elsewhere in the literature. Finally, a forecast of the persistent current effects to be expected in the LHC main dipoles is presented, and the more critical parameters for beam dynamics are singled out.
\end{abstract}

Index Terms-Accelerator magnets, LHC, magnetic field measurement, superconducting accelerator magnets, superconducting cables.

\section{INTRODUCTION}

$\mathbf{T}$ HE production of the Large Hadron Collider [1] superconducting main dipoles [2] is well under way, and has reached the nominal series production rate. One of the main elements of the magnet design is the high homogeneity of the magnetic field to ensure a stable motion of the circulating beams [3]. The steering of the dipole field quality toward the beam dynamics requirements is based on the measurements at room temperature (r.t.) and on their extrapolation to operational conditions using measurements at $1.9 \mathrm{~K}$ [4]. All magnets will be measured at room temperature and a sampling of $33 \%$ is foreseen at $1.9 \mathrm{~K}$ [5].

Room temperature measurements are extrapolated to operational conditions using a simple average on the warm-to-cold offsets over all magnets measured at $1.9 \mathrm{~K}$. These offsets depend at the injection energy on the magnetization properties of the cable, which may differ from a manufacturer to another manufacturer. Indeed, since the present sampling of magnets measured at $1.9 \mathrm{~K}$ does not reflect the final composition of the machine in terms of cable manufacturers, we could have a bias in the estimated offsets.

In this work we analyze the measurements of the cable magnetization [6], which is routinely done for all produced cables, focusing on differences between manufacturers.

Manuscript received October 3, 2004.

The authors are with CERN, European Organization for Nuclear Research, Accelerator Technology Department, Geneva, Switzerland (e-mail: Boris.Bellesia@cern.ch).

Digital Object Identifier 10.1109/TASC.2005.849534

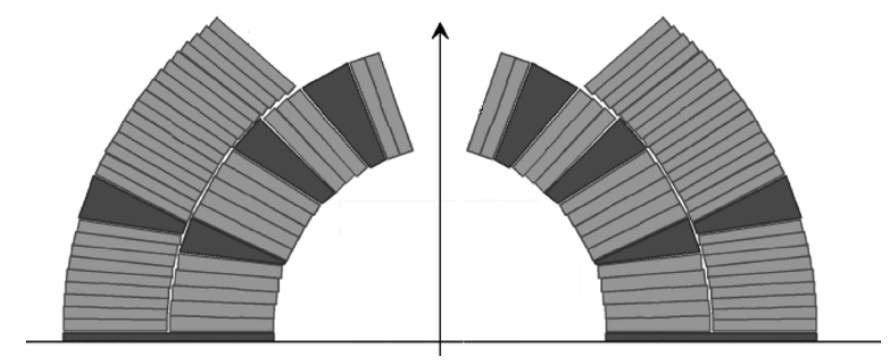

Fig. 1. Coil lay-out of the main dipole of the Large Hadron Collider (upper half of one aperture).

We then analyze the measured effect of persistent currents on field quality and its dependence on the cable manufacturers. The final aim is to compute impact of the cable manufacturer on the warm-to-cold offsets used for the steering of field quality.

\section{Magnetization Measurements of Cables}

\section{A. General Features of Cable Production}

The coil design of LHC dipole is based on a two layers $\cos \partial$ structure using two different Rutherford superconducting cables (see Fig. 1). The inner layer cable ("Cable01") is made of $28 \mathrm{NbTi}$ strands with $1.065 \mathrm{~mm}$ diameter; the outer layer cable ( "Cable02") is made of $36 \mathrm{NbTi}$ strands with $0.825 \mathrm{~mm}$ diameter. Before being accepted, cables have to satisfy control tests of magnetization, inter-strand cross contact resistance, copper to superconductor ratio, dimensional analysis, and critical current. More than $60 \%$ of the cables have been already manufactured; the production of Cable01 is shared by two firms (denoted by letters B and E) whilst that of Cable02 among five (B, C, D, G and $\mathrm{K})$. The quantities produced are such that for the Cable01, 5 octants are manufactured by B, and 3 by E. For the Cable 02 , 3 octants are manufactured by B, 2 by C, and 1 each by G, K and D.

\section{B. Cable Magnetization Measurements}

LHC strand magnetization is measured at CERN. The procedure of measurements is described in [6]. The specimen is put in an external field that is ramped up to $1 \mathrm{~T}$ and then is ramped down to the starting field at $1.9 \mathrm{~K}$; the magnetization curve is then obtained. Thereafter, the amplitude of the hysteresis of the magnetization curve at $0.5 \mathrm{~T}$ is evaluated ( $\Delta \mathrm{M}$ in Fig. 2). In order to avoid time dependent effects (eddy currents), additional measurements at different ramp rates of the field are performed 


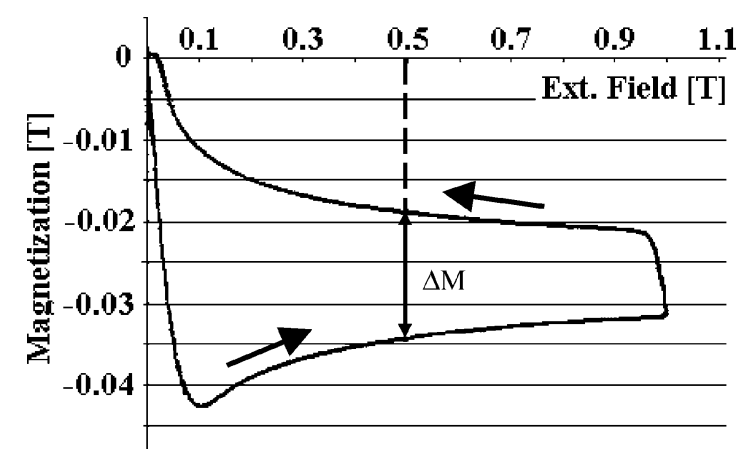

Fig. 2. Measured magnetization of a 01B strand and amplitude of the hysteresis loop at $0.5 \mathrm{~T}$ (external magnetic field ramp rate $8 \mathrm{mT} / \mathrm{s} ; 4.22 \mathrm{~K}$ ).

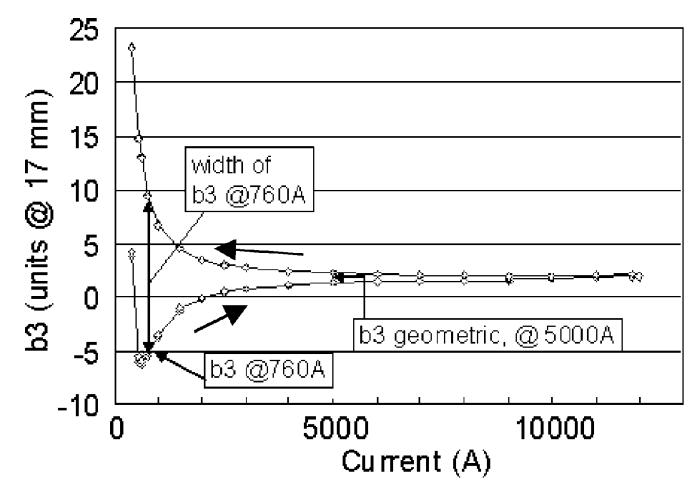

Fig. 3. Measured b3 along a standard loadline: width of the hysteresis at injection field and effective persistent current.

and the extrapolation to zero ramp-rate is taken. The cable magnetization is the average of the magnetizations of strands composing the cable.

The analysis of the measurements shows that the standard deviations of cable magnetization within each producer are around $4.5 \%$, thus indicating a very good quality control of the manufacturing processes. For Cable 01, the E manufacturer shows an average magnetization $13 \%$ larger than the one of B. For Cable02, $\mathrm{B}, \mathrm{G}$ and $\mathrm{K}$ have a very similar average, whereas $\mathrm{C}$ is $4.5 \%$ larger and $\mathrm{D}$ is $3.5 \%$ smaller. Most of the differences in magnetization can be traced back to the different design of the strands: $01 \mathrm{E}$ and $02 \mathrm{C}$ are based on a double stack layout whereas all other strands are manufactured via the single stack method.

\section{Persistent Current Measurements in the Dipoles}

\section{A. Measurements Procedure and Measured Magnets}

The measurements at $1.9 \mathrm{~K}$ of the magnetic field quality are performed following a so-called "loadline curve": the current is ramped from $350 \mathrm{~A}$ up to $12 \mathrm{kA}$ and then down to $350 \mathrm{~A}$ by successive steps. The intervals at constant current between steps last approximately $40 \mathrm{~s}$ in order to perform the measurement in a static condition, avoiding all transient effects. At each step, a complete measurement is performed. A pre-cycle from $350 \mathrm{~A}$ to $11850 \mathrm{~A}$ and then to $350 \mathrm{~A}$ is performed before measurements. Dynamic effects are measured separately, using a real machine cycle with a ramp rate of $10 \mathrm{~A} / \mathrm{s}$ : in this way one can separate the static from the dynamic contributions.

The measurement of a typical field harmonic obtained during the loadline is shown in Fig. 3. For each harmonic and for the
TABLE I

Cable Manufacturers of Measured Magnets

\begin{tabular}{lccc}
\hline \hline Cable & Number of magnets & Cable & Number of magnets \\
\hline 01B-02B & 35 & 01B-02C & 6 \\
01B-02K & 32 & 01E-02K & 11 \\
\hline
\end{tabular}

TABLE II

Measured Hysteresis at $1.9 \mathrm{~K}$ (MEAN AND STANDARD DEVIATION-UNITS @ 17 mm)

\begin{tabular}{ccccccccc}
\hline \hline & \multicolumn{2}{c}{ 01B-02B } & \multicolumn{2}{c}{ 01B-02C } & \multicolumn{2}{c}{$01 \mathrm{~B}-02 \mathrm{C}$} & \multicolumn{2}{c}{$01 \mathrm{E}-02 \mathrm{~K}$} \\
\hline & $\mu$ & $\sigma$ & $\mu$ & $\sigma$ & $\mu$ & $\sigma$ & $\mu$ & $\sigma$ \\
b1 & 10.1 & 2.6 & 13.1 & 1.6 & 9.1 & 3.4 & 4.6 & 2.7 \\
b3 & 14.9 & 0.4 & 15.8 & 0.8 & 14.8 & 0.3 & 14.7 & 0.5 \\
b5 & 1.87 & 0.13 & 1.78 & 0.08 & 1.78 & 0.06 & 2.26 & 0.15 \\
b7 & 0.70 & 0.05 & 0.79 & 0.02 & 0.69 & 0.03 & 0.91 & 0.06 \\
\hline
\end{tabular}

TABLE III

Measured Effective Persistent Current at $1.9 \mathrm{~K}$ (Mean and STANDARD DEVIATION-UNITS @ $17 \mathrm{~mm}$ )

\begin{tabular}{ccccccccc}
\hline \hline & \multicolumn{2}{c}{ 01B-02B } & \multicolumn{2}{c}{ 01B-02C } & \multicolumn{2}{c}{ 01B-02K } & \multicolumn{2}{c}{$01 \mathrm{E}-02 \mathrm{~K}$} \\
\hline & $\mu$ & $\sigma$ & $\mu$ & $\sigma$ & $\mu$ & $\sigma$ & $\mu$ & $\sigma$ \\
b1 & -1.57 & 1.41 & -2.01 & 1.71 & -0.35 & 1.55 & -0.58 & 1.63 \\
b3 & -7.25 & 0.34 & -7.52 & 0.32 & -6.88 & 0.30 & -6.77 & 0.37 \\
b5 & 1.13 & 0.12 & 1.12 & 0.08 & 1.03 & 0.06 & 1.2 & 0.07 \\
b7 & -0.32 & 0.04 & -0.35 & 0.04 & -0.31 & 0.04 & -0.40 & 0.04 \\
\hline
\end{tabular}

main field component one evaluates the width of the hysteresis at the injection field level $(760 \mathrm{~A})$. Moreover, the geometric component is defined as the average of measurements at $5 \mathrm{kA}$ obtained during ramping up and ramping down the current, and the so-called effective persistent current is defined as the value at injection field minus the geometric component during the ramp up (see Fig. 3). The effective persistent current is the only one relevant for the beam, which is ejected before the ramp down.

In this report, we present data of 84 measured magnets. In Table I we list the composition of the cable manufacturers for these magnets. A large statistics is available for the combination $01 \mathrm{~B}-02 \mathrm{~B}$, and $01 \mathrm{~B}-02 \mathrm{~K}$, whereas cable $01 \mathrm{E}$ is measured on 11 magnets only, and no data is available for cables $02 \mathrm{G}$ and $02 \mathrm{D}$, which have been used in magnets not yet measured at $1.9 \mathrm{~K}$.

\section{B. Measured Hysteresis}

In Table II we give for each cable configuration the average and the standard deviations of the widths of the hysteresis curves at $760 \mathrm{~A}$. There is a difference between cable 01B and 01E in the main field (cable $\mathrm{E}$ has 4.5 units less) and in higher order multipoles (cable $\mathrm{E}$ has $25 \%$ more hysteresis both in b5 and in b7). The differences in higher order multipoles, mainly affected by inner cable properties, can be explained by a different magnetization observed between cable 01B and 01E. Negligible differences are found for $b 3$. There is a significant contribution of the outer layer to the main field.

\section{Measured Effective Persistent Current}

The same analysis is performed in Table III for the so-called effective persistent component, i.e. the measurement at the injection field minus the geometric (see Fig. 3). This is the rele- 


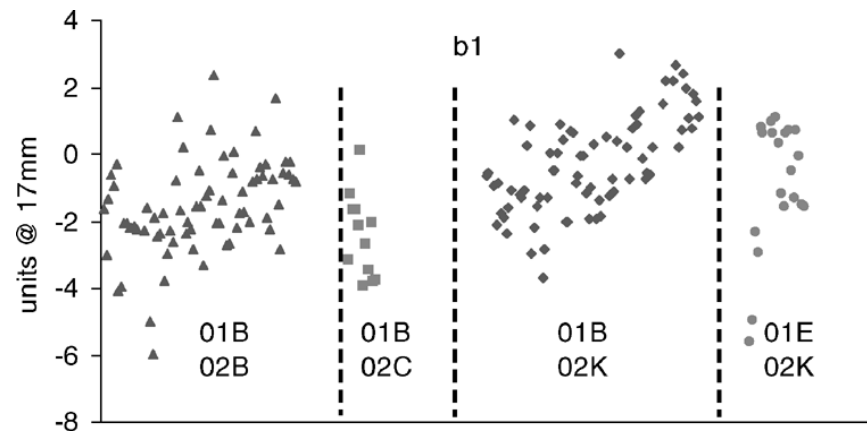

Fig. 4. Measured effective persistent current component versus combination of cable manufacturers for main field.

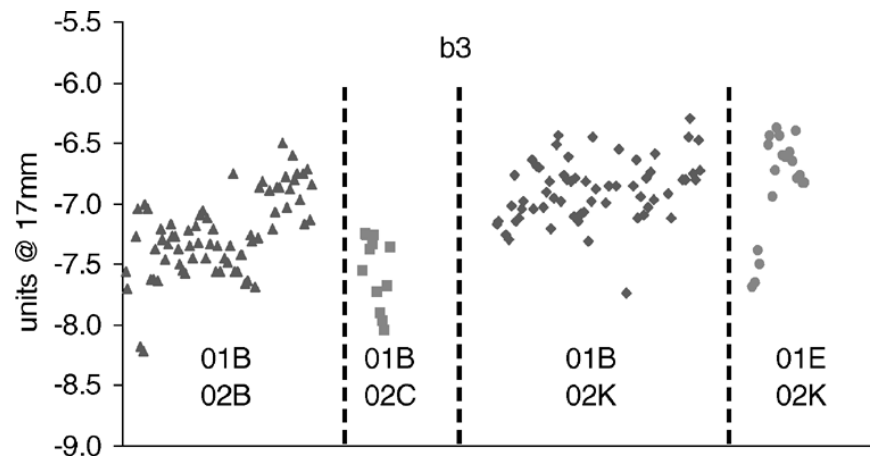

Fig. 5. Measured effective persistent current component versus combination of cable manufacturers for b3.

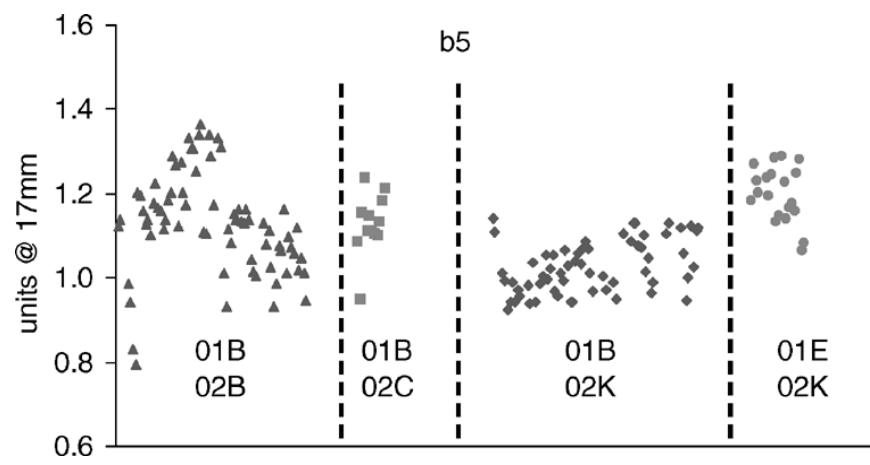

Fig. 6. Measured effective persistent current component versus combination of cable manufacturers for b5.

vant value for the beam. With respect to the hysteresis results, the difference between cable B and cable $\mathrm{E}$ in $\mathrm{b} 1$ is wiped out, and is reduced to $10 \%$ in b5 and to $20 \%$ in b7. This is due to the fact that the main difference in the hysteresis cycle between $B$ and $\mathrm{E}$ is in the branch with the ramp down of the current, which does not affect the effective persistent current component. Plots for b1, b3 and b5 are shown in Figs. 4, 5 and 6. The effective persistent current component is approximately half of the hysteresis, with the exception of b1, where not all the contribution comes from persistent currents.

\section{Persistent Currents Evaluated Through Models}

At this stage of the measurements (84 dipoles measured at $1.9 \mathrm{~K}$ ), one should rely on measurements of field quality rather than on models. Indeed, for cables $02 \mathrm{D}$ and $02 \mathrm{G}$ no measurements of persistent currents are available. On the other hand, the magnetization measurement of the strands show that these

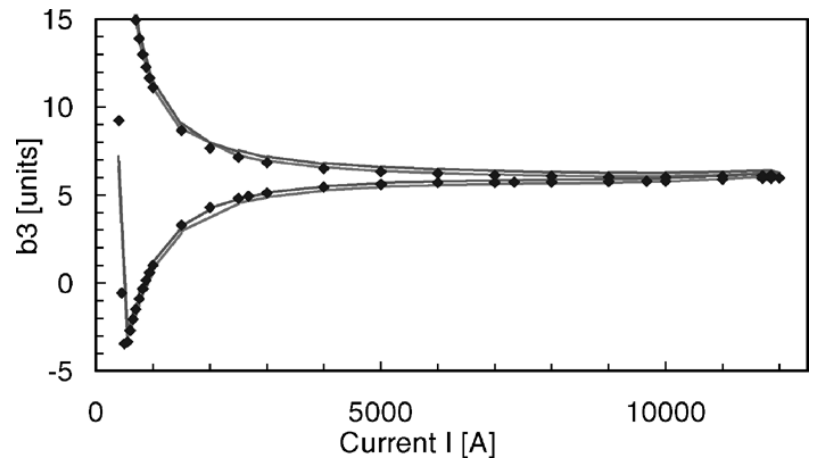

Fig. 7. Measured b3 versus excitation current (solid lines) and model based on magnetization measurements (dots).

cables do not show significant anomalies with respect to $02 \mathrm{C}$, $02 \mathrm{~B}$ and $02 \mathrm{~K}$ that have been measured in magnets.

Different models to evaluate persistent currents from magnetization have been developed. Here we have used one described in [9], using as input a fit function for the critical current density Jc that can be obtained from the magnetization measurement on the strand. The individual strand magnetization depends on the locally applied field and thus on the position within a coil cross-section. With respect to previous models, here the repercussion of the persistent current field on the source field in the coil has been taken into account by an iteration cycle, as the original field changes when the persistent current field is superposed.

A verification of the results of the model for a measured magnet is shown in Fig. 7. The agreement is very good for both hysteresis branches, all over the domain of interest (i.e. from 760 to $11850 \mathrm{~A}$ ). This model has then been used to compute the combination of cables not yet measured in magnets. As expected, the spread in main field and multipoles is not worse than what is presented in Table III.

\section{Dependence of the Beam Dynamics Targets at Room TEMPERATURE ON THE CABLE MANUfACTURER}

The average of multipoles over all the magnets of the machine (i.e., the so-called systematic) must be within the beam dynamics ranges [3]. Two constraints have to be satisfied in operational conditions at $1.9 \mathrm{~K}$ : at injection field and at high field. On the other hand, the steering of the field quality is based on the measurements at room temperature, and therefore injection and high field targets are projected to room temperature through the warm-to-cold correlations. Here, we use the measurements and models presented in the previous sections to determine the influence of cable manufacturer on the warm-to-cold correlations, and therefore on targets at r.t.

The easiest case is b7, since we only have a target of $[-0.3,0.1]$ units imposed at injection field. This range is projected at $r$. t. measurements by subtracting the beam screen contribution, the effective persistent current, and the variation of geometry due to cool-down, giving $[0.29,0.69]$ units (see Fig. 8, right). Changes in the effective persistent current due to different cable manufacturers can move the range at room temperature up to 0.09 units (see Tables III and IV). The effect is not negligible, as the width of the range is 0.4 units. 

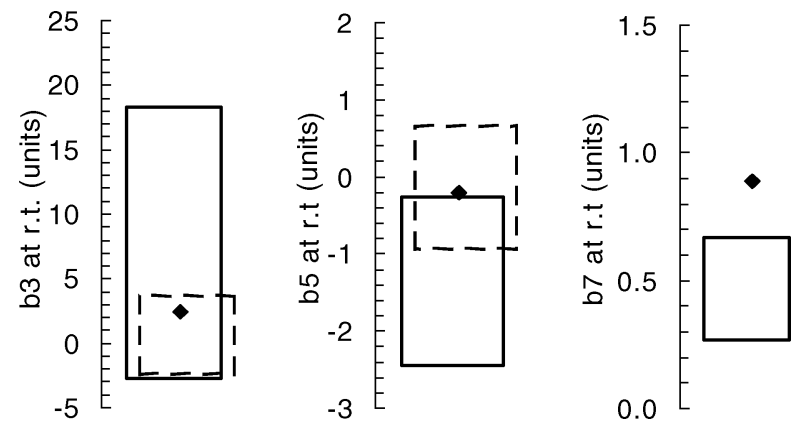

Fig. 8. Target ranges for systematic at room temperature for b3, b5 and b7: injection field constraints (solid lines) and high field constraints (dotted lines), and present measured values in the production (dots).

TABLE IV

BeAm Dynamics TARgets on MEASUREMENTS AT RoOM TEMPERATURE, MaX. AND Min. Evaluated w.r.t. THE CABle MANUFACTURER

\begin{tabular}{|c|c|c|c|c|}
\hline & \multicolumn{2}{|c|}{ Lower limit (units) } & \multicolumn{2}{|c|}{ Upper limit (units) } \\
\hline \multirow{3}{*}{ b3 } & \multicolumn{2}{|c|}{$\begin{array}{l}\text { Determined by injection } \\
\text { and high field }\end{array}$} & \multicolumn{2}{|c|}{ Determined by high field } \\
\hline & Min & Max & \multirow{2}{*}{\multicolumn{2}{|c|}{3.60}} \\
\hline & -2.40 & -2.29 & & \\
\hline \multirow{3}{*}{ b5 } & \multirow{3}{*}{\multicolumn{2}{|c|}{$\begin{array}{l}\text { Determined by high field } \\
-0.94\end{array}$}} & \multicolumn{2}{|c|}{ Determined by injection } \\
\hline & & & Min & Max \\
\hline & & & -0.35 & -0.18 \\
\hline \multirow{3}{*}{ b7 } & \multicolumn{2}{|c|}{ Determined by injection } & \multicolumn{2}{|c|}{ Determined by injection } \\
\hline & Min & Max & Min & Max \\
\hline & 0.25 & 0.34 & 0.65 & 0.74 \\
\hline
\end{tabular}

Systematic b5 must be within $[-1.1,1.1]$ units at injection field and within $[-0.8,0.8]$ units at high field. When the ranges are projected at room temperature, the injection target is shifted down by about 1.1 units, which is mainly due to the persistent current contribution (see Table III). On the other hand, there is a small difference between the high field target at $1.9 \mathrm{~K}[-0.8,0.8]$ and at room temperature, since the contributions of beam screen, cool down, saturation and Lorentz forces are small. The resulting intersection of the two ranges at r.t. (see Fig. 8, centre) shows that the lower limit is determined by the high field target and therefore it does not depend on the cable manufacturer. On the other hand, the upper limit is determined by injection field limit, and therefore depends on the effective persistent current. It can range between -0.35 and -0.18 units according to the cable manufacturer (see Table IV). This shift of 0.17 units is not negligible with respect to the width of the range ( 0.6 units). The present measured value is just on the edge of this value.

Systematic b3 at $1.9 \mathrm{~K}$ must be within [-3,3] units at high field and within $[-10.5,10.5]$ units at injection. Also in this case, the difference between high field range and r.t. range is small, whereas the injection field range is shifted up by around 7 units, mainly due to the persistent current contribution (see
Fig. 8, left). The high field range is included within the injection range as long as the offset between injection and high field is less than $10.5-3=7.5$ units. Since this offset is mainly due to the persistent current, this condition is satisfied for most of the cable manufacturers. This is why only a fraction of the variation in b3 effective persistent current shown in Table III is found in the variation of the lower limit in Table IV.

We conclude that the impact of cable manufacturer on the allowed ranges for the systematics at r.t. is about $30 \%$ of the width of the range for $\mathrm{b} 5,20 \%$ for $\mathrm{b} 7$, and negligible for $\mathrm{b} 3$.

\section{CONCLUSION}

The analysis of magnetization measurements of $50 \%$ of the LHC cables has been presented: differences in average magnetization between cable manufacturers are negligible, except for the case of the two producers of the inner cable (13\% difference between averages). Measurements of persistent current effects on field quality at $1.9 \mathrm{~K}$ in 84 magnets have been analyzed. The different cable magnetization of the inner layer producers induces different behaviors for high order multipoles (b5, b7, ..), and for the main field b1. This effect is well visible in the width of the hysteresis of main field and multipoles, whereas it is less evident in the part of the branch corresponding to the positive ramp of the field (the so-called effective persistent component), which is the only relevant for the beam.

The global impact of the different cable manufacturers on the persistent current component is not negligible for b5 and b7, and very small for b3. It should be taken into account when defining the extrapolation of the beam dynamics targets at room temperature, used for the steering of the production.

\section{REFERENCES}

[1] P. Lebrun, "Industrial technology for unprecented energy and luminosity: the Large Hadron Collider," in EPAC 2004, Lucerne, Switzerland, Jul. 05-09, 2004, also as LHC Project Report 745.

[2] L. Rossi, "Experience with LHC magnets from prototyping to large scale industrial production and integration," in EPAC 2004, Lucerne, Switzerland, Jul. 05-09, 2004, also as LHC Project Report 730.

[3] S. Fartoukh and O. Bruning, "Field quality specification for the LHC main dipole magnets," LHC Project Report 501, 2001.

[4] E. Todesco et al., "Steering field quality in the main dipole magnets of the Large Hadron Collider," IEEE Trans. Appl. Supercond., vol. 14, pp. 177-180, Jun. 2004.

[5] L. Bottura et al., "A strategy for sampling the field quality of the LHC dipoles," in EPAC 04.

[6] J. D. Adam et al., "Status of the LHC superconducting cable production," IEEE Trans. Appl. Supercond., vol. 12, pp. 1056-1062, Mar. 2002.

[7] R. Wolf and S. Le Naour, "The expected persistent current field errors in the LHC main dipole and quadrupole," LHC Project Note 230, 2001.

[8] L. Bottura, M. Schneider, L. Walckiers, and R. Wolf, "Cable magnetization effects in the LHC main dipole magnets," Adv. Cryog. Eng., vol. 43, pp. 451-458, Mar. 1998.

[9] M. Aleksa et al., "A vector hysteresis model for superconducting filament magnetization in accelerator magnets," in 14th Conf. Computation of Electromagnetic Fields, Saratoga Springs, USA, 2003. 